\title{
Phosphoenolpyruvate Carboxykinase in Experimental Intrauterine Growth Retardation in Rats
}

\author{
ARNOLD POLLAK, JOHN B. SUSA, BARBARA S. STONESTREET, ROBERT SCHWARTZ, AND \\ WILLIAM OH \\ Departments of Pediatrics, Women and Infants Hospital of Rhode Island, and Rhode Island Hospital, Section of \\ Reproductive and Developmental Medicine, Brown University Program in Medicine, Providence, Rhode Island, USA
}

\begin{abstract}
Summary
This study examines the role of impaired gluconeogenesis in the pathogenesis of neonatal hypoglycemia in intrauterine growth retardation (IUGR). IUGR was produced experimentally in eight pregnant rats by ligation of uterine arteries at the 17th day of gestation. Delivery occurred spontaneously at term. Sham operations were performed in five pregnant rats at the same gestational age and the fetuses delivered at term served as controls. The body weights of newborn rats with IUGR were significantly lower than the controls (5.32 \pm 0.12 vs. $6.22 \pm 0.06 \mathrm{~g}$, mean $\pm \mathrm{SE}, P<0.001$ ). The fetal liver weights were also significantly smaller in IUGR than in the control animals $(0.224 \pm 0.14$ vs. $0.340 \pm 0.12 \mathrm{~g}, P<$ 0.001). The activity of phosphoenolpyruvate carboxykinase (PEPCK) (the rate-limiting enzyme of gluconeogenesis) in liver cytosols was significantly lower in rats with IUGR $(0.06 \pm 0.01 \mathrm{vs.}$ $0.11 \pm 0.02 \mu \mathrm{M}$ phosphoenolpyruvate/g liver/min when compared with controls $(P<0.05)$. A direct relationship between this enzyme and the brith weight was observed, suggesting a close relationship between intrauterine nutrition and the status of gluconeogenesis. The blood glucose level was also lower in growth-retarded fetuses (36.6 \pm 4.7 vs. $69.6 \pm 4.3 \mathrm{mg} / \mathrm{dl}, \mathrm{p}<0.001$ ) when compared with controls. The data suggest that gluconeogenesis is impaired in IUGR and is partly responsible for the increase in the incidence of neonatal hypoglycemia in this group of subjects.
\end{abstract}

\section{Speculation}

Neonatal hypoglycemia in IUGR is probably caused by a combination of rapid depletion of glycogen stores and impaired gluconeogenesis, the latter indicated by a decreased liver PEPCK activity. This may be related to a diminished enzyme synthesis on the basis of significant energy and oxygen deprivation.

Recovery from postnatal fall in blood glucose concentration in full-term newborn rats is accomplished by glycongenolysis $(3,4)$ and developing gluconeogenesis $(2,9,17)$. In neonatal rats the latter is associated with an 8- to 10-fold rise of PEPCK activity (EC 4.1.32), the rate-limiting enzyme of gluconeogenesis in the liver cytosol, at 4-6 hr of age (16). A failure of these metabolic adaptations may result in hypoglycemia in different species including man $(3,5,11,13,23)$. Infants with IUGR are prone to develop neonatal hypoglycemia (13) probably based on reduced liver glycogen $(15,19)$, body fat (6), relative increase in substrate utilization $(3,5)$, and fialure of gluconeogenic regulation $(11,14$, 22).

Ligation of uterine arteries in pregnant rats at late gestation serves as an experimental model for IUGR on the basis of uteroplacental circulatory insufficiency (21). It has been shown that liver glycogen content and blood glucose were significantly reduced in IUGR when compared to control animals (15). In this report, we studied PEPCK activity in liver cytosols of newborn rats with IUGR to establish its role in the pathogenesis of hypoglycemia in IUGR.

\section{MATERIALS AND METHODS}

Thirteen time-dated pregnant rats of the Sprague-Dawley strain, weighing between 200 and $250 \mathrm{~g}$ were the subjects of this study. At 17 of the 22 days of gestation, a midline laparotomy was performed under ether anesthesia. The bicornate uterus was exposed and, in eight rats, both uterine arteries were ligated to produce IUGR and, in five, sham operations were done to provide control subjects. The uterus was returned into the abdominal cavity and the laparotomy wound was closed. The rats were fed ad libitum with water and Purina rat chow. The pregnancy was allowed to continue until spontaneous vaginal delivery occurred. The delivery of the newborn rats was precisely timed and the newborns were separated from their mothers, fasted, and maintained at an ambient thermal environment of $37^{\circ}$. At $4 \mathrm{hr}$ of age, the fetuses were killed by decapitation and their livers were removed, weighed, and kept frozen at $-20^{\circ}$ for PEPCK determination.

Liver cytosol was prepared by homogenization of the thawed liver in nine volumes ( $w / v)$ of cold $0.25 \mathrm{M}$ sucrose/ $10 \mathrm{mM}$ triethanolamine, $\mathrm{pH} 7.5$, buffer with a power-driven Potter-Elvehjem homogenizer. The homogenate was centrifuged at 105,000 $\times g$ for $60 \mathrm{~min}$ and the cytosol was removed for assay for PEPCK by a modified method of Seubert and Huth (18).

The reaction mixture consisted of $50 \mathrm{mM}$ HEPES- $\mathrm{NaOH}, 2$ $\mathrm{mM}$ inosine triphosphate, $3 \mathrm{mM} \mathrm{MgCl}_{2}, 1 \mathrm{mM}$ reduced glutathione, and $1.5 \mathrm{mM}$ oxaloacetate in a total volume of $1.0 \mathrm{~mL}$. The enzymatic reaction was terminated after $30 \mathrm{~min}$ at $25^{\circ}$ by the addition of $12 \mathrm{mg}$ tetraethylammonium tetrahydriodoborate in $0.1 \mathrm{ml}$ of $7.5 \mathrm{mM} \mathrm{NaOH}$.

Blood glucose was determined in 16 newborn rats with IUGR and 14 controls by glucose oxidase method with a Y. S. I. (24) glucose analyzer. The samples were obtained from axillary vessels according to Girard et al. (7). The $t$ test and method of leastsquares were used for statistical analysis.

\section{RESULTS}

As shown in Table I, body and liver weights of the IUGR groups were significantly smaller than in controls $(P<0.001)$. Blood glucose was also significantly lower in rats with IUGR (range, $12-64 \mathrm{mg} / \mathrm{dl})(P<0.00 \mathrm{l})$. A significant direct correlation between blood glucose and birth weight was noted in the experimental group ( $r=0.85, P<0.001$ ) (Fig. 1). No such correlation was found in the control animals. The blood glucose of the latter group of animals ranged from $36-95 \mathrm{mg} / \mathrm{dl}$. PEPCK activity in 
the liver cytosol was significantly lower in rats with IUGR than in control animals $(P<0.05)$. When the enzyme activity was expressed as per unit body weight to represent total body gluconeogenesis, the significant difference in PEPCK activity between IUGR and controls is even more apparent (2.32 \pm 1.57 vs. 4.91 \pm 1.67 nmole phosphoenolpyruvate $/ \mathrm{g}$ body wt/min, $P<0.001$ ) A direct linear correlation between birth weight and PEPCK activity was also noted in both groups of animals (Fig. 2). There was no difference between the two regression lines, indicating that IUGR alone has no effect on the PEPCK activity and that the birth weight is the primary determinant for its relationship with PEPCK activity.

When the mean data of body weights, liver weights, blood glucose, and PEPCK activities from each litter were analyzed to eliminate the biologic variability encountered among and within litters, the result still showed similar statistical significance between the IUGR and control groups.

\section{DISCUSSION}

In the present study, body and liver weights are significantly reduced in the experimental compared to the control fetuses. These data confirm the validity of the model in producing IUGR by reducing uteroplacental blood flow and fetal nutritional supply. It has been demonstrated in other studies that the reduction of fetal body weight as well as visceral organ weights is a valid index for IUGR in this model $(12,15)$.

Table 1. Birth weight, liver weight, and PEPCK activity in IUGR rats $^{1}$

\begin{tabular}{|c|c|c|}
\hline & IUGR & Controis \\
\hline No. of maternal rats & 8 & 5 \\
\hline No, of fetuses & 47 & 39 \\
\hline Birth wejght (g) & $5.32 \pm 0.12$ & $6.22 \pm 0.06^{2}$ \\
\hline $\begin{array}{l}\text { Fetal liver wet weight (g) } \\
\text { PEPCK }\end{array}$ & $0.224 \pm 0.014$ & $0.340 \pm 0.012^{2}$ \\
\hline $\begin{array}{c}\text { ( } \begin{array}{c}\text { mole } \mathrm{PEP} / \mathrm{g} \\
\text { liver } / \mathrm{min})\end{array}\end{array}$ & $0.06 \pm 0.01$ & $0.11 \pm 0.02^{3}$ \\
\hline $\begin{array}{l}\text { (nmole PEP/g body } \\
\text { wt/min) }\end{array}$ & $2.32 \pm 1.57$ & $4.91 \pm 1.67^{2}$ \\
\hline Blood glucose $(\mathrm{mg} / \mathrm{dl})$ & $36.6 \pm 4.7(16)^{4}$ & $69.6 \pm 4.3^{2}(14)$ \\
\hline
\end{tabular}

\footnotetext{
'Values are mean $\pm \mathrm{SE}$.

${ }^{2} P<0.001$.

${ }^{3} P<0.05$.

${ }^{4}$ Number of blood glucose determinations in parentheses.
}

\section{BLOOD GLUCOSE (mg/d)}

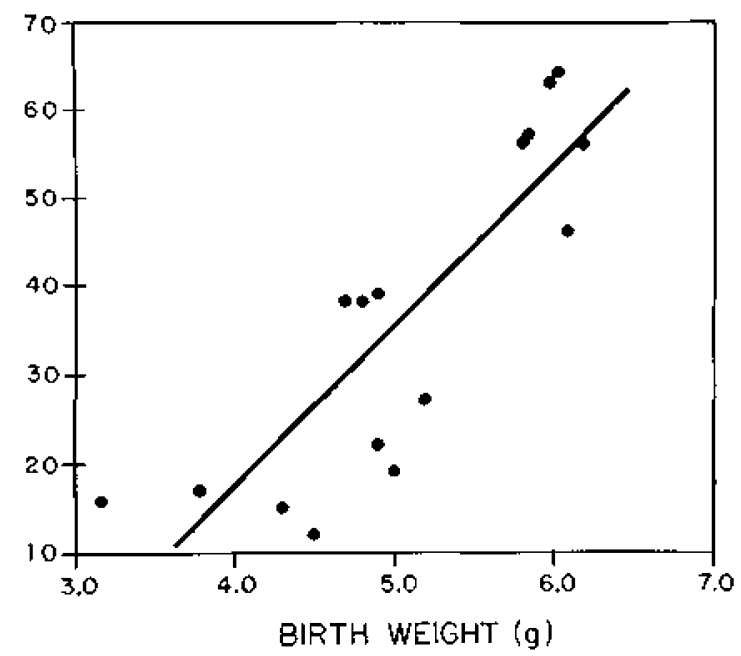

Fig. I. Relationship between blood glucose and body weight in 16 newborn rats with IUGR at $4 \mathrm{hr}$ old.
PEPCK ( $\mu$ moles PEP/g Liver/min)

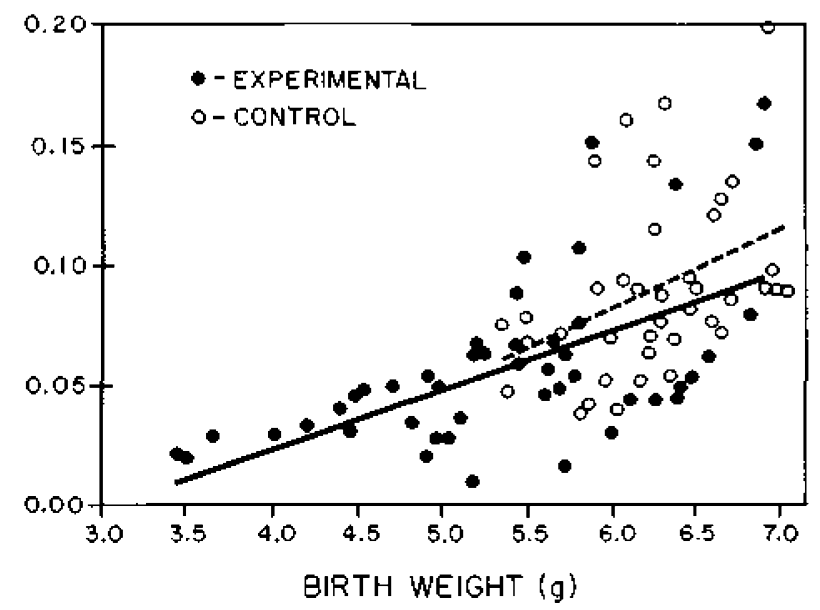

Fig. 2. Relationships between body weight and PEPCK activity in liver cytosols of newborn rats with IUGR and control animals at $4 \mathrm{hr}$ old. The solid line indicates the regression line for the experimental group; the dotied line indicates the regression line for the control group.

The birth weights in IUGR ranged from 3.4-6.9 and from 5.3$7.1 \mathrm{~g}$ in the control fetuses. The considerable overlap in birth weight in both groups can be explained as follows: In the IUGR group, the most growth-retarded fetuses (smallest birth weights) probably represent those located in the segment of the uterus supplied by the ovarian arteries. Previous work on the same model $(12,21)$ confirmed this concept. The variation in birth weight within the control fetuses suggests that, even in the normal gestation, there is variation in growth.

It has been shown that in full-term rats a dramatic increase in PEPCK activity and gluconeogenesis can be measured at $4 \mathrm{hr}$ of age, at which time the postnatal fall in blood glucose begins to level off $(7,9,16)$. Gluconeogenesis in the newborn rat is controlled by a number of factors including the redox state of the liver $(I, 15)$, the availability of amino acids $(8,15)$, as well as the activity of PEPCK. The rapid increase in gluconeogenesis from amino acids and pyruvate (20) that roughly parallels the rapid rise in PEPCK activity suggests that PEPCK plays a pivotal role in the control of gluconeogenesis.

In our study, the activity per gram liver of cytosolic PEPCK was directly related to the birth weight (Fig. 2). Although this relationship holds true for both groups, it is most apparent in the IUGR fetuses. Because birth weight partly reflects the nutritional status in utero, one may speculate that a poor nutritional status associated with low PEPCK activity may result in decreased gluconeogenic capacity. This concept is further suggested by our observation that the regression lines depicting the relationship between PEPCK activities and body weight are similar between control and IUGR groups. Using a similar rat model, Nitzan and Groffmann (14) also demonstrated that glucose production de novo from ${ }^{14} \mathrm{C}$-labeled alanine was significantly lower in IUGR rats. Assuming that glycogenolysis and gluconeogenesis are important adaptive processes for the normalization of blood glucose in the first few hours of life in rats, it can be postulated that a decrease in gluconeogenesis during this time may play an important role for the pathogenesis of hypoglycemia in IUGR.

It should be pointed out that extrapolation of the animal IUGR model to human infants in this problem is always far from ideal and may be inappropriate. However, studies by Williams et al. (22) using oral alanine-loading techniques demonstrated similarly inefficient gluconeogenic capabilities in human newborn infants with IUGR when compared with normals.

The reason for decreased PEPCK activity in IUGR remains unclear. It has been shown previously that total body protein content of IUGR rats was significantly reduced (12); one may speculate that the lower PEPCK activity in the experimental 